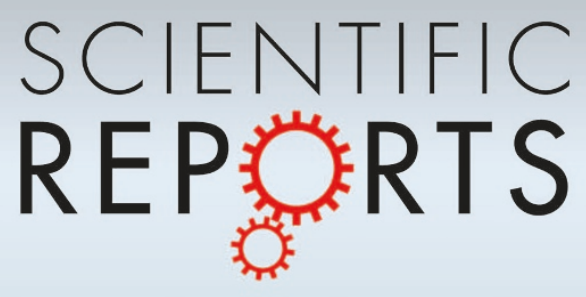

OPEN

SUBJECT AREAS:

SYNTHESIS AND

PROCESSING

MECHANICAL PROPERTIES

POLYMERS

GELS AND HYDROGELS

Received

30 May 2013

Accepted

10 September 2013

Published

3 October 2013

Correspondence and requests for materials should be addressed to

S.C. (chensu@njut. edu.cn)

\section{Robust Self-Healing Hydrogels Assisted by Cross-Linked Nanofiber Networks}

\author{
Yuan Fang, Cai-Feng Wang, Zhi-Hong Zhang, Huan Shao \& Su Chen
}

State Key Laboratory of Materials-Oriented Chemical Engineering and College of Chemistry and Chemical Engineering, Nanjing University of Technology, Nanjing 210009, P. R. China.

Given increasing environmental and energy issues, mimicking nature to confer synthetic materials with self-healing property to expand their lifespan is highly desirable. Just like human skin recovers itself upon damage with the aid of nutrient-laden blood vascularization, designing smart materials with microvascular network to accelerate self-healing is workable but continues to be a challenge. Here we report a new strategy to prepare robust self-healing hydrogels assisted by a healing layer composed of electrospun cross-linked nanofiber networks containing redox agents. The hydrogels process high healing rate ranging from seconds to days and great mechanical strengths with storage modulus up to $0.1 \mathrm{MPa}$. More interestingly, when the healing layer is embedded into the crack of the hydrogel, accelerated self-healing is observed and the healing efficiency is about $\mathbf{8 0 \%}$. The healing layer encourages molecular diffusion as well as further cross-linking in the crack region of the hydrogel, responsible for enhanced healing efficiency.

S elf-healing is a key biological feature that prolongs the lifespan of living organisms $s^{1-3}$. On human skin, for instance, a cut is able to heal spontaneously, where blood vascularization enables the delivery of nutrients for this recovery. Given increasing environmental and energy issues, mimicking nature to confer synthetic materials with self-healing property to expand their lifetime is highly desirable ${ }^{4-8}$. Specifically, artificial microvascular systems have been created by some elaborate techniques such as soft lithography ${ }^{9,10}$ or direct-write assembly ${ }^{11}$. And Sottos' group incorporated the microvascular networks into epoxy coating to realize self-healing $^{12-16}$. Meanwhile, three-dimensionally cross-linked hydrogels with self-healing ability have aroused increasing interest owing to their potentials in diverse biomedical application ${ }^{17-24}$. In this system, self-healing usually occurs through the introduction of reversible covalent bonds ${ }^{18-20}$, or non-covalent supramolecular interactions such as hydrogen bonds $s^{21}, \pi-\pi$ interactions $s^{22}$, host-guest interaction $s^{23}$ or hydrophobic interaction $s^{24}$. Despite great progress in this field, new facile strategies to endow hydrogels with combination of self-healing and mechanical toughness are still highly needed ${ }^{17}$.

Herein, we developed cross-linked nanofiber networks filled with redox agents to mimic nutrient-laden blood vascularization to speed up the healing process of hydrogels with intrinsic self-healing capability. The hydrogels, prepared by using biomedical materials poly (1-vinyl-2-pyrrolidinone) (PVP) modified with O-carboxymethyl chitosan $(\mathrm{O}-\mathrm{CMC})$ and acrylamide $(\mathrm{AAm})$, can bear external forces owing to their great mechanical strength and efficiently and autonomically heal themselves following damage, representing the rare examples of synthetic materials with combination of intrinsic self-healing capability and mechanical toughness ${ }^{24}$. Moreover, inspired by healing process in human skin where damage can heal spontaneously with the aid of nutrient-laden blood vascularization, we designed a healing layer (a bilayer film) composed of electrospun cross-linked nanofiber networks to mimic blood vascularization, with oxidant and reductant loaded in separated layer to serve as nutrients (healing agents). The healing layer with cross-linked nanofiber networks could release and deliver redox agents efficiently through capillary force after embedded into the cracks of hydrogels, initiating further cross-linking reaction to rebond two hydrogel interfaces into an integrated one. Then, halved healing time without compromising mechanical properties of the hydrogels was realized. Importantly, the healing layer has the same composition as the bulk hydrogels, which can avoid chemical contamination and offer good affinity and hence endow high healing efficiency to the healing materials. This new facile strategy that employing electrospun cross-linked nanofiber networks containing redox agents mimic nutrient-laden blood vascularization to enhance self-healing performance of materials avoids the complex fabrication process required for self-healing microvascular systems, might contributing a technically simple and easy handling way to fabricate self-healing materials with useful applications. 

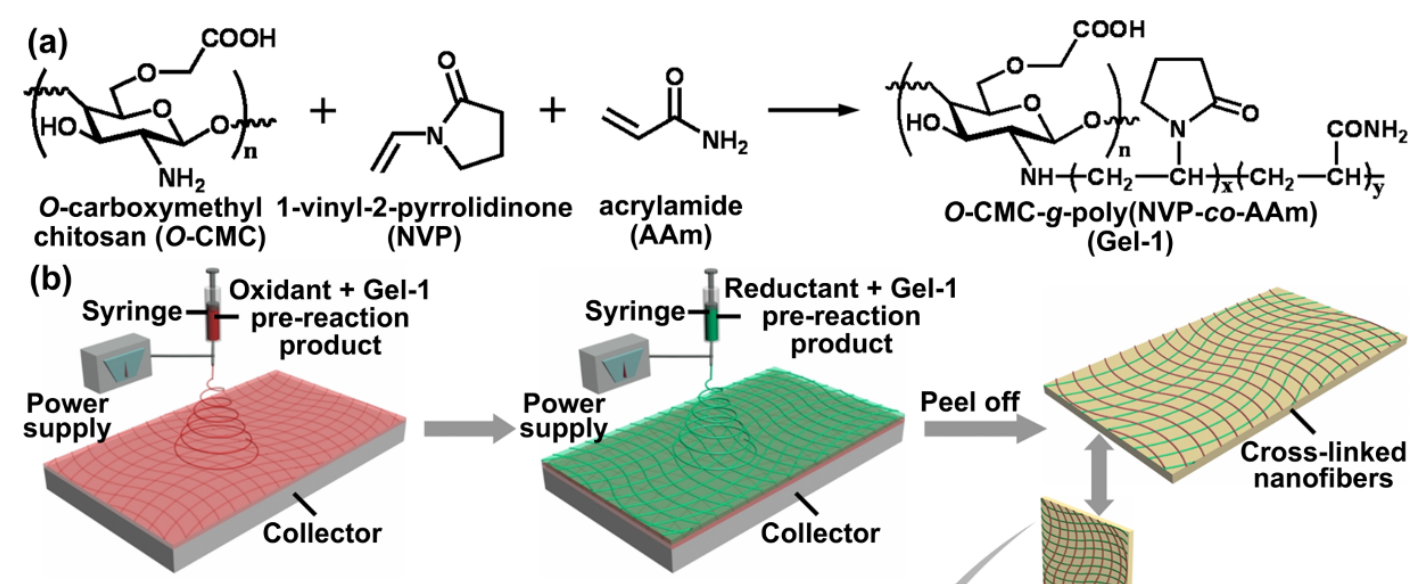

(Gel-1)
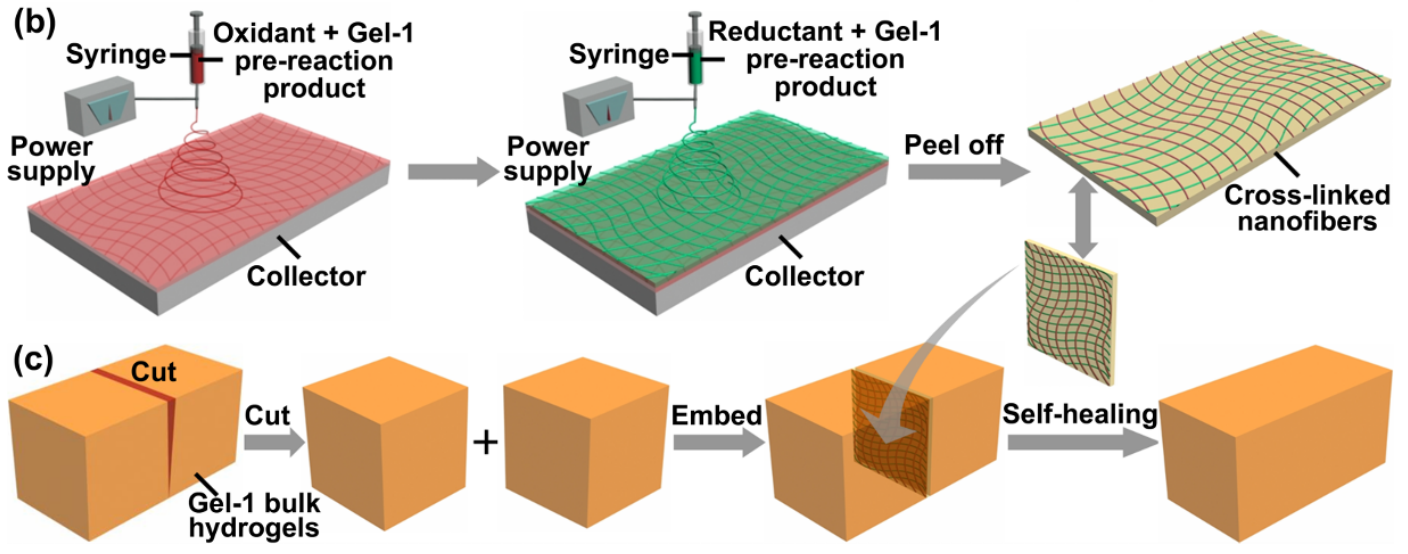

Figure 1 Schematic preparation and enhanced self-healing procedure of Gel-1. (a) Synthetic scheme of Gel-1. (b) Fabrication of Gel-1 healing layer formed of nanofiber networks. (c) Enhanced self-healing procedure upon embedding a healing layer into the damage area of Gel-1 bulk hydrogels.

\section{Results}

The synthetic scheme and enhanced healing procedure for selfhealing hydrogels is shown in Fig. 1. As shown in Fig. 1a, the hydrogels O-CMC-g-poly(NVP-co-AAm) (referred to as Gel-1, where NVP = 1-vinyl-2-pyrrolidinone) were prepared from free radical copolymerization of $O-C M C, N V P$, and AAm. O-CMC has been employed in wound healing due to its excellent biocompatibility and adhesive properties. PVP has good biocompatibility and low toxicity, but poor mechanical strength limiting its potential application. Here we chose the cross-linking monomer AAm as the copolymerization monomer to address this issue. To fabricate Gel-1 bulk hydrogels, the ammonium persulfate (APS) $/ N, N, N^{\prime}, N^{\prime}$-tetramethylethylenediamine (TEMED) couple were used as the initiator. The redox reaction of APS and TEMED can give two types of primary radical species from amine and persulfide (Supplementary Fig. S1), initiating cross-linking reaction and profiting the formation of hydrogel networks ${ }^{25-27}$. To enhance healing performance of hydrogels, an electrospinning technique, a cost-effective approach for facile generation of nano-/microfibrous polymers ${ }^{28-32}$, was employed to prepare a cross-linked nanofiber bilayer film filled with both oxidant and reductant as a healing layer. As shown in Fig. 1b, an aqueous solution of Gel-1 pre-reaction product containing oxidant APS was electrospun into nanofibers on a copper wire mesh collector, followed by the same implement for a Gel-1 pre-reaction product solution containing reductant TEMED, affording a bilayer film composed of a layer of Gel-1 nanofibers loaded with oxidant APS and the other layer of Gel-1 nanofibers loaded with reductant TEMED. Then, the Gel-1 bulk hydrogel was cut into two separated blocks, followed by embedment of the healing layer, to obtain a sandwich-structural hydrogel (Fig. 1c). Without any external intervention, these pieces merged into an integrated one much more quickly than in the situation without the healing layer.

The morphologies and properties of Gel-1 hydrogels were thoroughly investigated. Scanning electron microscopy (SEM) measurements reveal cross-linked porous structure of Gel-1 bulk hydrogels (Fig.s 2a,b). As the ratio of [AAm]/[NVP] increases, the pore size of the hydrogel decreases, and so does its swelling capacity (Supplementary Fig. S6). Fig. $2 c$ shows the storage modulus $\left(G^{\prime}\right)$ and loss modulus $\left(G^{\prime \prime}\right)$ of the hydrogels as the functions of frequency at a fixed strain of $0.01 \%$. The $G^{\prime}$ values show a substantial elastic response and are larger than the $G^{\prime \prime}$ values over the entire range of frequencies. Both moduli increase after introduction of small amount of cross-linker BIS $(0.02 \mathrm{wt} \%)$. It is noted that the $G^{\prime}$ value can be up to $0.1 \mathrm{MPa}$, comparable to the largest value reported for self-healing hydrogels ${ }^{17}$, suggesting that the hydrogels have high mechanical strengths. Strain sweeps shown in Fig. $2 \mathrm{~d}$ reveal that the $G^{\prime}$ value decreases and is slightly smaller than the $G^{\prime \prime}$ value above the critical strain region of $0.42 \%$, indicating a collapse of the gel state. Furthermore, the hydrogel can be stretched from $7.0 \mathrm{~cm}$ to $23.0 \mathrm{~cm}$, and then shrunk back to $7.1 \mathrm{~cm}$ without any visible damage, indicating that it can be recovered rapidly (Fig.s $2 \mathrm{e}-\mathrm{g}$ ). The results demonstrate that Gel-1 hydrogels have great mechanical strength and recover capability.

The self-healing behavior of Gel-1 hydrogels is shown in Fig. 3. When the bulk hydrogel is cut into separated pieces by a razor blade and then juxtaposed in contact, they will merge into an integrated one after a certain time without any external intervention. The effect of various preparation parameters on the healing rate of self-healing hydrogels was investigated, as shown in Fig.s 3a-c and Supplementary Table S1. As the ratio of [AAm]/[NVP] or the content of cross-linker BIS increases, the healing rate of the hydrogels decreases (Fig.s 3a,b). As shown in Fig. 3a, the hydrogels prepared from $[\mathrm{AAm}] /[\mathrm{NVP}]=1: 1,2: 1$, and $3: 1 \mathrm{~mol} / \mathrm{mol}$, respectively, show healing time of 1 day (d), $2 \mathrm{~d}$, and $3 \mathrm{~d}$, respectively. These results are consistent with the previous report that the chemical hydrogels with high cross-link density lack the ability to self-heal ${ }^{33}$. In addition, chitosan is a bioadhesive molecule that it can strongly bind surrounding tissue through hydrogen bonding. In our case, the increase in $\mathrm{O}-\mathrm{CMC}$ concentration leads to the increase in the healing rate of the hydrogels (Fig. 3c). This indicates that the presence of chitosan increases the healing rate, which is in good agreement with the results in the literature ${ }^{34}$. Specifically, when the hydrogel prepared from $\mathrm{NVP}=0.04 \mathrm{~mol}$, BIS $=0.02 \mathrm{wt} \%$, and $O-\mathrm{CMC} /$ monomer $=$ $2.5 \%(\mathrm{w} / \mathrm{w})$ was cut by a blade, it spontaneously healed within $15 \mathrm{~s}$ (Fig.s 3d-f; Supplementary Movie S1). The width of the wound upon fresh cut was $119.2 \mu \mathrm{m}$ (Fig. $3 \mathrm{~d}$ ), and then became $105.2 \mu \mathrm{m}$ after $5 \mathrm{~s}$ (Fig. 3e). With increasing time, the wound disappeared fast, and the damaged areas self-healed completely within $15 \mathrm{~s}$ (Fig. 3f).

Significantly, accelerated self-healing was observed after the healing layer composed of electrospun cross-linked nanofiber networks with redox agents was embedded into the damaged area of hydrogels (Fig.s $1 \mathrm{c}$ and $3 \mathrm{a}-\mathrm{c}$ ). For example, as the healing layer was introduced 

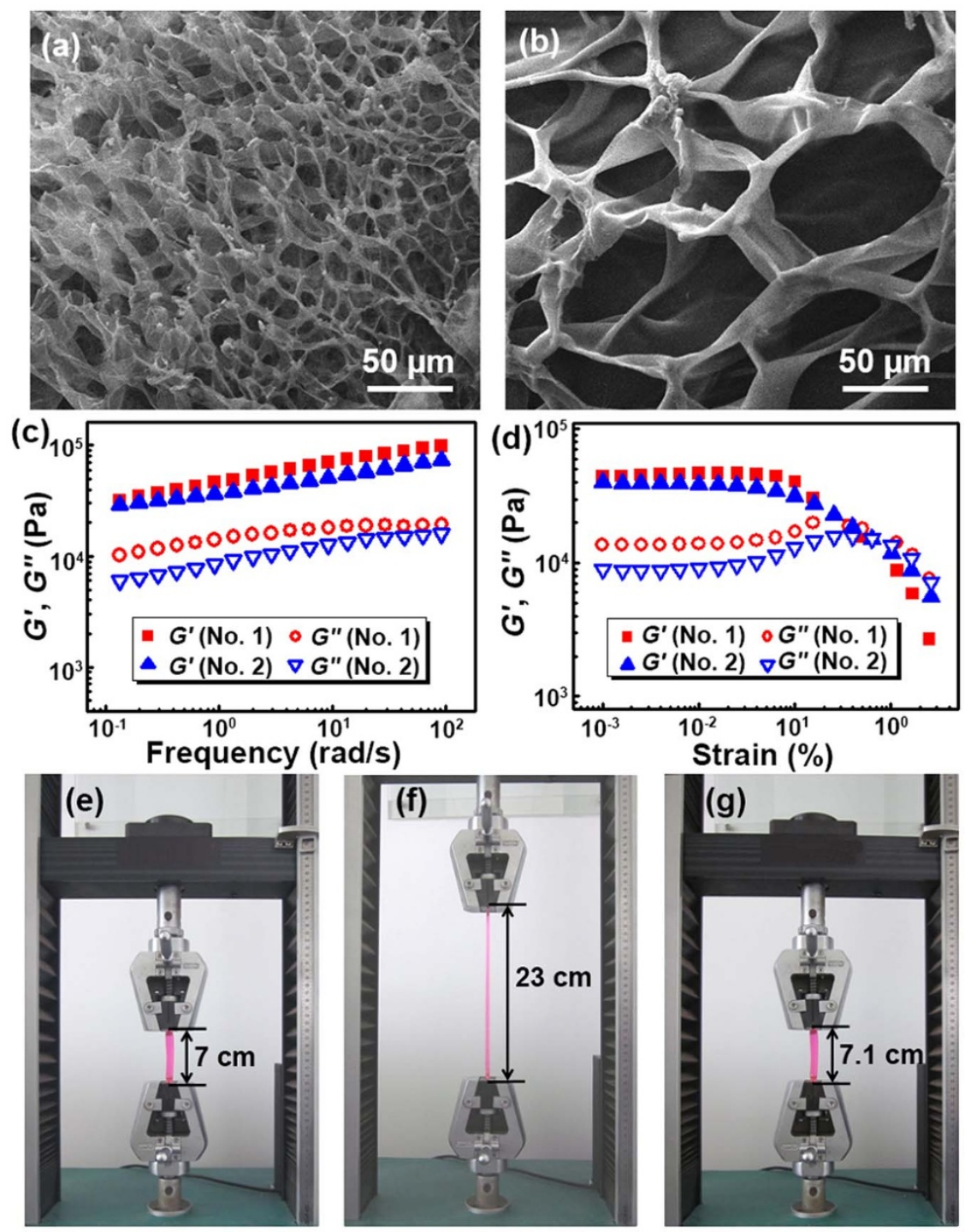

Figure $2 \mid$ Morphology and mechanical properties of hydrogels. (a,b) SEM images of hydrogels with $O-\mathrm{CMC} / \mathrm{monomer}=2.5 \%(\mathrm{w} / \mathrm{w}), \mathrm{BIS}=0.02 \mathrm{wt} \%$ at different $[\mathrm{AAm}] /[\mathrm{NVP}]$ ratios of a) $3: 1$ and b) $1: 2(\mathrm{~mol} / \mathrm{mol})$. (c,d) Storage modulus $\left(G^{\prime}\right)$ and loss modulus $\left(G^{\prime \prime}\right)$ values of the hydrogels on (c) frequency sweeps and $(\mathrm{d})$ strain sweeps: $[\mathrm{AAm}] /[\mathrm{NVP}]=3: 1(\mathrm{~mol} / \mathrm{mol}), \mathrm{O}-\mathrm{CMC} / \mathrm{monomer}=2.5 \%(\mathrm{w} / \mathrm{w})$, and BIS $=0.02 \mathrm{wt} \%$ for No.1 and BIS $=$ $0 \mathrm{wt} \%$ for No.2. (e-g) Stretching and shrinking processes of the hydrogel (No. 1) dopped with 0.05 wt $\%$ rhodamine B during the tensile measurements: (e) the original hydrogel, (f) the hydrogel in elongation, and ( $\mathrm{g}$ ) the hydrogel after elongation.

into the cracks of the hydrogels prepared from $[\mathrm{AAm}] /[\mathrm{NVP}]=3: 1$, $2: 1$, and $1: 1 \mathrm{~mol} / \mathrm{mol}$, respectively, the structural integrity of the corresponding hydrogels could be restored after $2 \mathrm{~d}, 1 \mathrm{~d}$, and $0.5 \mathrm{~d}$, respectively (Fig. 3a), noting that the embedment of the healing layer reduces half of the healing time for the hydrogels. This finding also accords with the other runs (Fig.s 3b,c, and Supplementary Table S1), indicating that the healing layer plays a vital role in enhancing healing rate. Moreover, as the healed cracks recut, the self-healing process occurred once again, suggesting this self-healing process could be repeatable.

To investigate the healing efficiency of the hydrogels upon embedding with the healing layers, we constructed a 7.0-cm-long column by juxtaposing hydrogel blocks (transparent), healing layer (not seen), and hydrogel blocks doped with rhodamine B (red) in sequence and alternately (Fig. $3 \mathrm{~g}$ ). Even though the blocks were not cut freshly, the healing layer easily stuck them together. The resulting column is very stable, even not damaged after bended by hands (Fig. 3h). Additionally, we connected together two $2.5 \mathrm{~cm}$-long blocks inserted with a healing layer in the same way (Fig. 3i). As shown in Fig. 3j, the joint between the two blocks in the merged sample was strong enough to sustain vigorous stretching. The tensile tests conducted on the original hydrogel gave an elongation ratio at break of $773 \pm$ $38 \%$ at $136.9 \pm 3 \mathrm{kPa}$, while those on the healing layer-embedded healed hydrogel gained an elongation ratio at break of $626 \pm 32 \%$ at $111.5 \pm 2 \mathrm{kPa}$ (Supplementary Table S2). The elongation ratio at break of the healed hydrogel is similar to the original one and the healing efficiency of this hydrogel is about $80 \%$. The results suggest that the introduction of the healing layer into the damaged area of hydrogels can highly accelerate self-healing without compromising mechanical properties of the hydrogels.

\section{Discussion}

What is the origin of the self-healing nature of Gel-1 hydrogels and the enhanced self-healing performance of hydrogels by embedding the healing layer? We propose that self-healing nature of Gel-1 hydrogels could be attributed to hydrogen bonding across two hydrogel pieces. As seen in Supplementary Fig. S7a, two Gel-1 hydrogel pieces can be merged into an integrated one through hydrogen bonding between amide, hydroxyl, and carboxylic functional groups. To testify our proposal, we immersed the self-healed Gel-1 hydrogels 

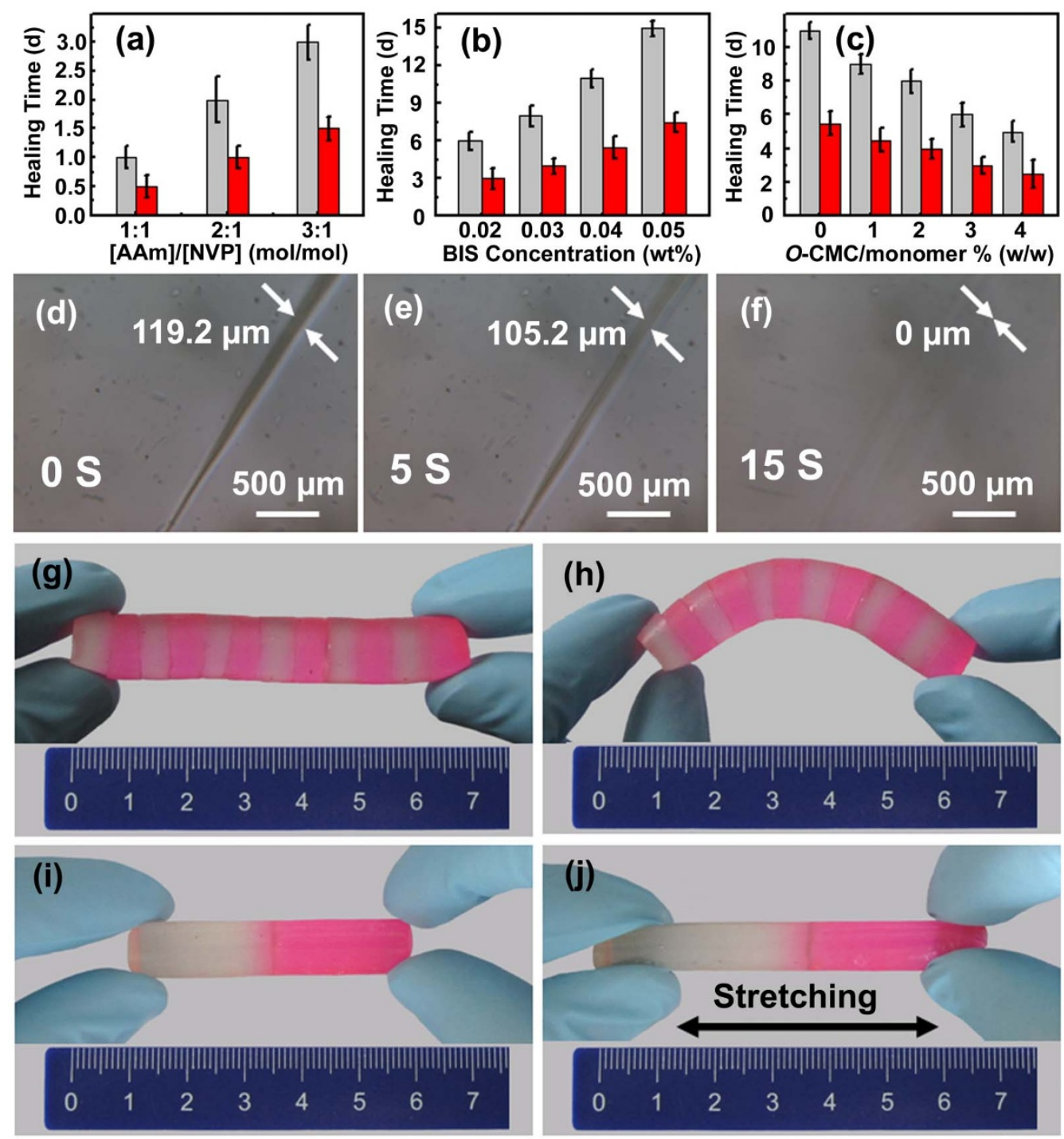

Figure 3 Self-healing properties of hydrogels. (a-c) The effect of preparation parameters on the healing rate of the hydrogels embedded with (red bars) and without (light gray bars) the healing layer, respectively. ( $\mathrm{d}-\mathrm{f}$ ) Optical images of the damaged hydrogel recorded as time after cut by a blade:

(d) $0 \mathrm{~s}$, (e) $5 \mathrm{~s}$, and (f) $15 \mathrm{~s}$. (g-j) A hydrogel column constructed from the connection of twelve hydrogel blocks alternating with six healing layers can be (g) held horizontally and (h) bended by hands, and a sample constructed by juxtaposing a healing layer between two hydrogel blocks exhibits extraordinary mechanical toughness: (i) before and (j) after stretching. Hydrogels with (red) and without (transparent) 0.05 wt $\%$ rhodamine B were prepared at $[\mathrm{AAm}] /[\mathrm{NVP}]=3: 1(\mathrm{~mol} / \mathrm{mol}), \mathrm{BIS}=0 \mathrm{wt} \%$, and $O-\mathrm{CMC} / \mathrm{monomer}=2.5 \%(\mathrm{w} / \mathrm{w})$ and cut into small blocks prior to use.

into an aqueous solution of $30 \%$ urea to disrupt the hydrogen bonds ${ }^{21,35}$. As expected, the two blocks of the self-healed Gel-1 hydogels were separated after immersed in the urea solution (Supplementary Fig. S7b).

We also explored the mechanism for enhanced self-healing performance of hydrogels by embedding the healing layer. Field emission scanning electron microscopy (FESEM) measurements show that the original Gel-1 pre-reaction product nanofibers have an average diameter of about $100 \mathrm{~nm}$ (Fig 4a, and Supplementary Fig.s S7, S8). Whereas, the nanofibers in the healing layer of bilayer film loaded redox agents display cross-linked network structure with increased diameter of $300 \mathrm{~nm}$ (Fig. 4b), which are further crosslinked and swollen with diameter of $c a .500 \mathrm{~nm}$ after embedded into the hydrogels (Fig. 4c). These features suggest the occurrence of cross-linking reaction initiated by APS and TEMED filled in the nanofibers. This result implies the presence of redox agents in the nano-network healing layer and their implement in cross-linking reaction to further form hydrogel networks. To further confirm this point, we prepared a sample coating with a healing layer composed of a nanofibrous bilayer film at the outside of the hydrogel and that without healing layer for comparison. Additionally, in order to explain the role of nano-networks, we fabricated a bilayer film without nanofiber structure using spin-coating method, with the same composition as the bilayer film with nanofiber structure, as a negative control. After immersed in Gel-1 pre-reaction product aqueous solutions containing $0.05 \mathrm{wt} \%$ rhodamine B for $1 \mathrm{~d}$, the sample coating with the nanofibrous bilayer film absorbed the most amount of the Gel-1 pre-reaction product solution, to form the most amount of new hydrogels around the original one (Supplementary Fig. S10). This experiment indicates the nano-network healing layer promotes the release of redox agents which initiate cross-linking reaction to further form more hydrogel networks.

Diffusion has been reported to be the most critical stage of crack healing process ${ }^{36,37}$, and we found that the introduction of the healing layer with cross-linked network into the hydrogels facilitates molecular diffusion. As shown in Fig.s 4d,e, two-block hydrogels with and without the healing layer embedded into the merged interface were prepared, respectively, where one hydrogel block in each kind of 

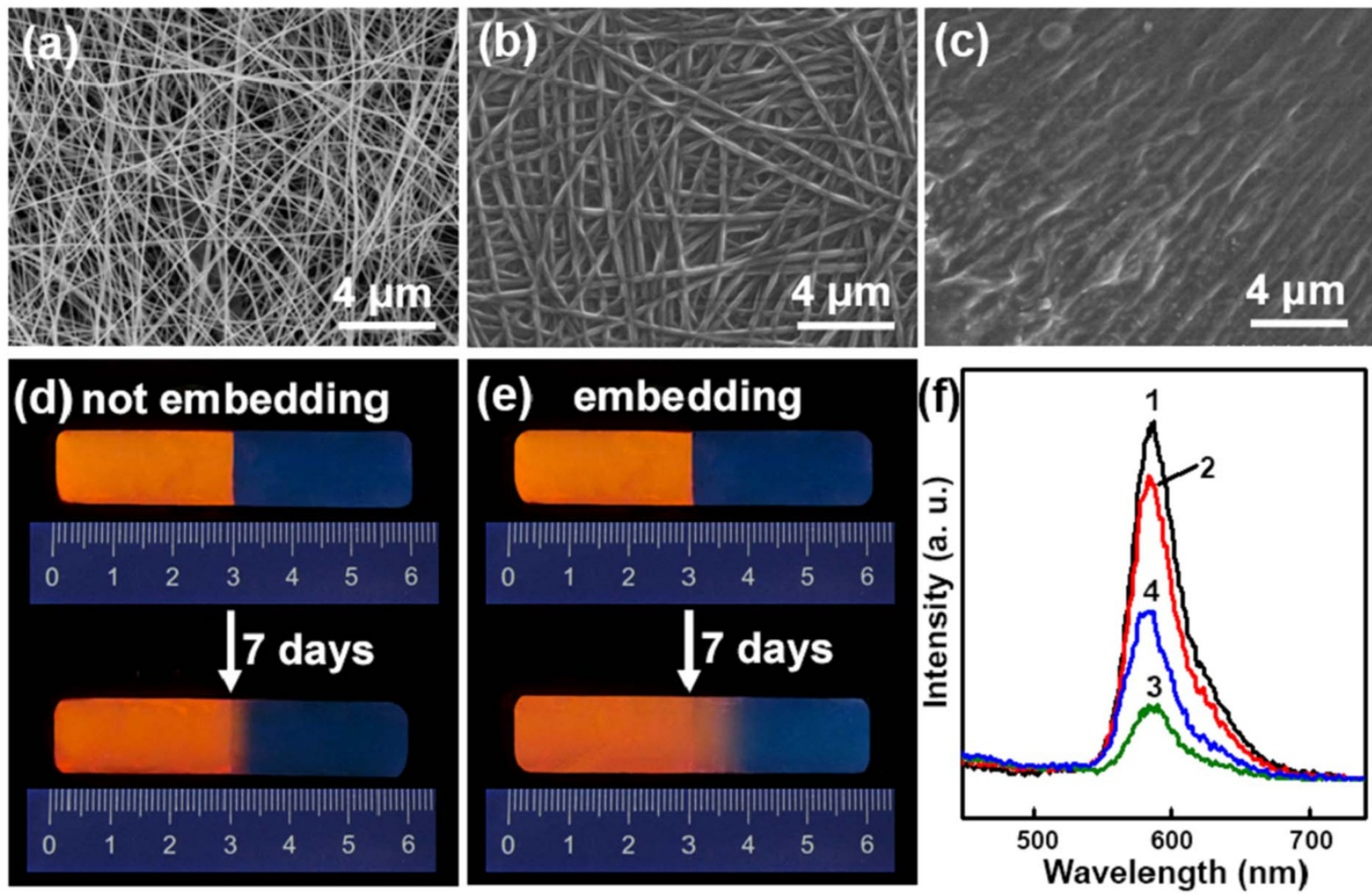

Figure $4 \mid$ Morphology and molecular diffusion process in healed hydrogels embedded with and without the healing layer. (a-c) FESEM images of (a) nanofibers without redox initiators, and the healing layer (b) as-prepared and (c) after embedding into bulk hydrogels. (d,e) Fluorescent images and (f) fluorescence spectra of hydrogels constructed by connecting together two hydrogel blocks with and without 0.05 wt $\%$ rhodamine B respectively stored for 7 days: (d) without and (e) with the healing layer embedded between the two merged hydrogel blocks. (f) The corresponding fluorescence spectra of hydrogels after stored for 7 days: at position of $(1,2) 2.8 \mathrm{~cm}$ and $(3,4) 3.2 \mathrm{~cm}$ for $(1,3)$ sample $(\mathrm{d})$ and $(2,4)$ sample (e).

healed hydrogels was doped with fluorescent dye marker rhodamine B. Upon UV excitation ( $\lambda=302 \mathrm{~nm}$ ), the hydrogel blocks with rhodamine $B$ show bright orange PL emission (580 nm), whereas the hydrogel blocks without rhodamine B exhibit blue PL emission $(380 \mathrm{~nm})$ (Fig.s 4d,e and Supplementary Fig. S11). After the healed hydrogels without healing layer stored for $7 \mathrm{~d}$, rhodamine B diffused from the position of $3 \mathrm{~cm}$ (the boundary of the two blocks) to the position of $3.2 \mathrm{~cm}$. Whereas, for the healed hydrogels embedded with healing layer, rhodamine B diffused to the position of $3.8 \mathrm{~cm}$. These reveal the molecular diffusion in the sample embedding with the healing layer is much faster than in the sample without healing layer. These diffusion features can also be confirmed by fluorescence spectra, where the sample embedding with the healing layer shows relatively lower fluorescent intensity at the position of $2.8 \mathrm{~cm}$ and higher fluorescent intensity at the position of $3.2 \mathrm{~cm}$ in comparison with those without healing layer, respectively (Fig. 4f). The above results indicate the use of electrospun cross-linked nanofiber networks with redox agents as healing layer to embed between two hydrogel blocks can accelerate the molecular diffusion from one hydrogel block to the other based on capillary force, through which redox agents can release efficiently and disperse across the interfaces of hydrogel pieces to initiate further cross-linking and rebond two hydrogel interfaces into an integrated one, and hence accelerated self-healing is achieved.

In conclusion, we synthesized O-CMC-modified poly(NVP-coAAm) self-healing hydrogels with high mechanical strength. By adjusting the preparation parameters in this system, the self-healing processes with healing rate ranging from seconds to days were available, which is meaningful for the application of self-healing hydrogels. Abundant hydrogen bonding in this cross-linked system might be responsible for the intrinsic self-healing capability of hydrogels.
Moreover, we developed a new facile method to accelerate the healing rate of hydrogels based on the introduction of electrospun crosslinked nanofiber networks containing redox initiators as the healing layer, mimicking nutrient-laden blood vascularization enabling spontaneous recovery of a cut in human skin. This approach shows the unique advance that cross-linked nanofibers profit the molecular diffusion along the boundary of the damaged blocks and the released redox agents initiate further cross-linking across the rupture, and thus reduce half of the healing time. Also importantly, the healing layer has the same composition as the bulk hydrogels, avioding chemical contamination to the healing materials. With healing efficiency up to $80 \%$, the mechanical strength of the healed hydrogels is similar to the original one. This work opens promising avenues to achieve the potential development of more appropriate self-healing hydrogels with electrospun cross-linked nanofiber networks, and thus might be adapted for more practical applications, for instance, in controlled drug delivery materials, regenerative medicines and wound dressings.

\section{Methods}

Materials. Acrylamide (AAm; 97\%), 1-vinyl-2-pyrrolidinone (NVP; 99\%), $N, N^{\prime}$ methylenebisacrylamide (BIS; 98\%), ethanol absolute (99.5\%), and $N, N, N^{\prime}, N^{\prime}$ tetramethylethylenediamine (TEMED; 99\%) were purchased from Sigma-Aldrich and used as received without any further purification. $O$-carboxymethyl chitosan $(O$ CMC) was obtained from Zhejiang AoXing Biotechnology Co. Ltd. (China) with a substitution degree of $0.8, M_{\mathrm{w}}$ of 9000 , and used without any further treatment. Ammonium persulfate (APS; 98\%) was ordered from Sigma-Aldrich and purified by recrystallization in distilled water before use. High-purity water with the resistivity of greater than $18 \mathrm{M} \Omega \cdot \mathrm{cm}^{-1}$ was used in the experiments.

Preparation of Gel-1 bulk hydrogels. The appropriate amounts of AAm, NVP, O$\mathrm{CMC}$, and APS were mixed in a certain amount of water to form a homogeneous solution in a container. A typical composition was water $=60 \mathrm{wt} \%,[\mathrm{AAm}] /[\mathrm{NVP}]=$ $3: 1(\mathrm{~mol} / \mathrm{mol}), O-C M C /$ monomer $=2.5 \%(\mathrm{w} / \mathrm{w})$, and APS $=0.04 \mathrm{wt} \%$. After the 
reductant TEMED ([APS] $/[$ TEMED] $=1: 4 \mathrm{~mol} / \mathrm{mol}$ ) was added and mixed homogenously, the solution was sealed in the container and placed at ambient temperature without stirring until the hydrogel was formed. After the completion of reaction, the samples were purified by immersing in deionized water for 7 days to remove all water-soluble materials. Then the samples were dried in a vacuum oven at $60^{\circ} \mathrm{C}$ until the weight of the specimen was constant.

Synthesis of Gel-1 pre-reaction products. Gel-1 pre-reaction products were prepared by aqueous free radical copolymerization of AAm and NVP with O-CMC using APS as an initiator in a four-necked round flask with a mechanical stirrer, a thermometer, a reflux condenser, and a nitrogen gas-introducing tube. A typical synthesis of Gel-1 pre-reaction products is as follows: A mixture of $5.63 \mathrm{~g} \mathrm{AAm}$ $2.93 \mathrm{~g} \mathrm{NVP}$ and $0.22 \mathrm{~g} \mathrm{O}$-CMC was dissolved in $40 \mathrm{~g}$ purified water in a $100 \mathrm{~mL}$ round flask, and the stirring speed was set at $250 \mathrm{rpm}$. The flask was immersed in an oil bath set at $70^{\circ} \mathrm{C}$. The reaction mixture was purged with nitrogen for $20 \mathrm{~min}$. Once the reaction temperature had been reached, polymerization was initiated by $10 \mathrm{~g}$ aqueous solution containing $0.04 \mathrm{~g}$ APS. The polymerization reaction was allowed to continue for $2 \mathrm{~h}$. The resulting viscous solution was then allowed to cool and was subsequently precipitated in an excess amount of ethanol and separated by centrifugation. The precipitate was washed with ethanol several times to remove the unreacted monomers, and then the precipitated Gel-1 pre-reaction products were dried in a vacuum oven at $60^{\circ} \mathrm{C}$ overnight.

Fabrication of electrospun cross-linked nanofiber networks. The electrospun apparatus used in this work consists of a high-voltage power, a syringe, an 18 gauge stainless steel needle and a grounded stainless steel plane collector (wrapped with a copper wire mesh) mounted on an insulating stand at a distance of $15 \mathrm{~cm}$ from the needle. The electrospinning solution was maintained at a feed rate of $0.5 \mathrm{~mL} / \mathrm{h}$ under a constant potential of $15 \mathrm{kV}$. The ambient temperature was $25^{\circ} \mathrm{C}$ and the humidity was $55 \%$. The cross-linked nanofiber networks contained two layers of nanofibers. The layer 1 was electrospun from a aqueous solution of Gel-1 pre-reaction product (12 wt $\%)$ containing oxidant APS $(0.1 \mathrm{wt} \%)$ on the copper wire mesh for $2.5 \mathrm{~h}$, and then the layer 2 was electrospun from a Gel-1 pre-reaction product aqueous solution (12 wt\%) containing reductant TEMED ( $0.2 \mathrm{wt} \%)$ over layer $1 \mathrm{for} 2.5 \mathrm{~h}$, affording a two-layered film composed of a layer of Gel-1 nanofibers loaded with oxidant APS and the other layer with Gel-1 nanofibers loaded with reductant TEMED. Finally, the obtained cross-linked nanofiber networks were peeled off from the copper wire mesh.

Characterizations. To further identify the chemical structures of the samples, fourier transform infrared spectra (FT-IR) were recorded on a Nicolet-6700 spectrometer with $\mathrm{KBr}$ pellets in the $400-4000 \mathrm{~cm}^{-1}$ region at a resolution of $4 \mathrm{~cm}^{-1}$ (32 scans). ${ }^{1} \mathrm{H}$ high-resolution magnetic-angle spinning (HR-MRS) NMR spectra were collected at $400 \mathrm{MHz}$ on a Bruker Avance 400D instrument. Molecular weight distributions were analyzed by Gel permeation chromatography (GPC, Waters Instruments) with an Ultrahydrogel column. The morphology of the electrospun hydrogel nanofibers was observed by field emission scanning electron microscopy (FESEM) with a Hitachi S4800 instrument at $20 \mathrm{kV}$. Photographic images of the samples were captured by digital camera (SX210 IS, Canon) or stereomicroscope (SZM45). The structures of the bulk hydrogels obtained were examined using a QUANTA 200 SEM (Philips-FEI, Holland) operated at $20.0 \mathrm{kV}$. The samples used for SEM measurement were immersed in distilled water after synthesis to remove the residual unreacted monomers. During this period, the distilled water was changed daily until the samples reached the maximum swelling ratios. The samples were then freeze-dried for $12 \mathrm{~h}$. Dried samples used for SEM measurement were cut to expose their inner structure. The swelling properties of the as-prepared bulk hydrogels were measured based on gravimetric analysis. The rheological experiments of the bulk hydrogels were conducted on a HAAKE RheoStress 600 instrument using a parallel plate of $20 \mathrm{~mm}$ diameter at $20^{\circ} \mathrm{C}$. Photoluminescence (PL) spectra were measured on a Varian Cary Eclipse spectrophotometer equipped with a Xe lamp at room temperature. The elongation measurements were performed on the cylindrical hydrogel samples using a test machine at $25^{\circ} \mathrm{C}$ under the following conditions: crosshead speed $=15 \mathrm{~mm} /$ min, sample length between jaws $=48 \mathrm{~mm}$. The tensile strength and percentage elongation at break were recorded. For reproducibility, at least six samples were measured for each hydrogel sample and the results were averaged.

1. Burnworth, M. et al. Optically healable supramolecular polymers. Nature 472, 334-337 (2011).

2. Zhang, M. Q. \& Rong, M. Z. Basics of Self-Healing: State of the Art. Self-Healing Polymers and Polymer Composites. 1-81 (John Wiley \& Sons, Hoboken, 2011).

3. Li, Y., Li, L. \& Sun, J. Q. Bioinspired self-healing superhydrophobic coatings. Angew. Chem. Int. Ed. 49, 6129-6133 (2010).

4. Andersson, H. M., Keller, M. W., Moore, J. S., Sottos, N. R. \& White, S. R. SelfHealing Polymers and Composites. Self Healing Materials. van der Zwaag, S. (ed.), 19-44 (Springer, Dordrecht, 2007).

5. Wei, Q. et al. Self-healing hyperbranched poly(aroyltriazole)s. Sci. Rep. 3, 1093; doi:10.1038/srep01093 (2013).

6. Cho, S. H., White, S. R. \& Braun, P. V. Room-temperature polydimethylsiloxanebased self-healing polymers. Chem. Mater. 24, 4209-4214 (2012).

7. Ghosh, B. \& Urban, M. W. Self-repairing oxetane-substituted chitosan polyurethane networks. Science 323, 1458-1460 (2009).
8. Gupta, S., Zhang, Q. L., Emrick, T., Balazs, A. C. \& Russell, T. P. Entropy-driven segregation of nanoparticles to cracks in multilayered composite polymer structures. Nat. Mater. 5, 229-233 (2006).

9. Choi, N. W. et al. Microfluidic scaffolds for tissue engineering. Nat. Mater. 6, 908-915 (2007)

10. Runyon, M. K., Johnson-Kerner, B. L., Kastrup, C. J., Van Ha, T. G. \& Ismagilov, R. F. Propagation of blood clotting in the complex biochemical network of hemostasis is described by a simple mechanism. J. Am. Chem. Soc. 129, 7014-7015 (2007).

11. Therriault, D., White, S. R. \& Lewis, J. A. Chaotic mixing in three-dimensional microvascular networks fabricated by direct-write assembly. Nat. Mater. 2, 265-271 (2003).

12. Toohey, K. S., Sottos, N. R., Lewis, J. A., Moore, J. S. \& White, S. R. Self-healing materials with microvascular networks. Nat. Mater. 6, 581-585 (2007).

13. Hansen, C. J. et al. Self-healing materials with interpenetrating microvascular networks. Adv. Mater. 21, 4143-4147 (2009).

14. Hansen, C. J., White, S. R., Sottos, N. R. \& Lewis, J. A. Accelerated self-healing via ternary interpenetrating microvascular networks. Adv. Funct. Mater. 21, 4320-4326 (2011).

15. Esser-Kahn, A. P. et al. Three-dimensional microvascular fiber-reinforced composites. Adv. Mater. 23, 3654-3658 (2011).

16. Hamilton, A. R., Sottos, N. R. \& White, S. R. Self-healing of internal damage in synthetic vascular materials. Adv. Mater. 22, 5159-5163 (2010).

17. Wang, Q. et al. High-water-content mouldable hydrogels by mixing clay and a dendritic molecular binder. Nature 463, 339-343 (2010).

18. Deng, G. H., Tang, C. M., Li, F. Y., Jiang, H. F. \& Chen, Y. M. Self-healing of thermoplastics via living polymerization. Macromolecules 43, 1191-1194 (2010).

19. Deng, G. H. et al. Dynamic hydrogels with an environmental adaptive self-healing ability and dual responsive sol-gel transitions. ACS Macro Lett. 1, 275-279 (2012).

20. Zhang, Y. L., Tao, L., Li, S. X. \& Wei, Y. Synthesis of multiresponsive and dynamic chitosan-based hydrogels for controlled release of bioactive molecules. Biomacromolecules 12, 2894-2901 (2011).

21. Phadke, A. et al. Rapid self-healing hydrogels. PNAS 109, 4383-4388 (2012).

22. Yoshida, M. et al. Oligomeric electrolyte as a multifunctional gelator. J. Am. Chem. Soc. 129, 11039-11041 (2007).

23. Nakahata, M., Takashima, Y., Yamaguchi, H. \& Harada, A. Redox-responsive selfhealing materials formed from host-guest polymers. Nat. Commun. 2, 511-516 (2011).

24. Tuncaboylu, D. C., Sari, M., Oppermann, W. \& Okay, O. Tough and self-healing hydrogels formed via hydrophobic interactions. Macromolecules 44, 4997-5005 (2011).

25. Gu, Z. et al. Injectable Nano-Network for Glucose-Mediated Insulin Delivery. ACS Nano 7, 4194-4201 (2013).

26. Fang, Y., Yu, H., Chen, L. \& Chen, S. Facile glycerol-assisted synthesis of $N$-vinyl pyrrolidinone-based thermosensitive hydrogels via frontal polymerization. Chem. Mater. 21, 4711-4718 (2009).

27. Guo, X., Wang, C. F., Fang, Y., Chen, L. \& Chen, S. Fast synthesis of versatile nanocrystal-embedded hydrogels toward the sensing of heavy metal ions and organoamines. J. Mater. Chem. 21, 1124-1129 (2011).

28. Bognitzki, M. et al. Nanostructured fibers via electrospinning. Adv. Mater. 13, 70-72 (2001)

29. Yang, S. Y., Wang, C. F. \& Chen, S. A release-induced response for the rapid recognition of latent fingerprints and formation of inkjet-printed patterns. Angew. Chem. Int. Ed. 50, 3706-3709 (2011).

30. Li, D. \& Xia, Y. N. Electrospinning of nanofibers: Reinventing the wheel? Adv. Mater. 16, 1151-1170 (2004).

31. Greiner, A. \& Wendorff, J. H. Electrospinning: A fascinating method for the preparation of ultrathin fibres. Angew. Chem. Int. Ed. 46, 5670-5703 (2007).

32. Park, J. H. \& Braun, P. V. Coaxial electrospinning of self-healing coatings. $A d v$ Mater. 22, 496-499 (2010).

33. Peppas, N. A., Huang, Y., Torres-Lugo, M., Ward, J. H. \& Zhang, J. Physicochemical foundations and structural design of hydrogels in medicine and biology. Annu. Rev. Biomed. Eng. 2, 9-29 (2000).

34. Jin, Q., Schexnailder, P., Gaharwar, A. K. \& Schmidt, G. Silicate Cross-linked bionanocomposite hydrogels from $\mathrm{PEO}$ and chitosan. Macromol. Biosci. 9, 1028-1035 (2009)

35. McQueen-Mason, S. \& Cosgrove, D. J. Disruption of hydrogen bonding between plant cell wall polymers by proteins that induce wall extension. PNAS 91 6574-6578 (1994).

36. Wool, R. P. Self-healing materials: a review. Soft Matter 4, 400-418 (2008).

37. South, A. B. \& Lyon, L. A. Autonomic Self-Healing of Hydrogel Thin Films. Angew. Chem. Int. Edit. 49, 767-771 (2010).

\section{Acknowledgments}

This work was supported by the National High Technology Research and Development Program of China (863 Program) (2012AA030313), National Natural Science Foundation of China (21076103 and 21006046), National Natural Science Foundation of China-NSAF (10976012), and Priority Academic Program Development of Jiangsu Higher Education Institutions (PAPD). 


\section{Author contributions}

S.C. conceived the project and designed the experiments. Y.F., Z.-H.Z. and H.S. performed the experiments and analyzed the data. Y.F., C.-F.W. and S.C. contributed to the interpretation of the results and wrote the paper.

\section{Additional information}

Supplementary information accompanies this paper at http://www.nature.com/ scientificreports
Competing financial interests: The authors declare no competing financial interests.

How to cite this article: Fang, Y., Wang, C., Zhang, Z., Shao, H. \& Chen, S. Robust Self-Healing Hydrogels Assisted by Cross-Linked Nanofiber Networks. Sci. Rep. 3, 2811; DOI:10.1038/srep02811 (2013)

(c) (i) $\odot$ This work is licensed under a Creative Commons Attribution-

cc. visit http://creativecommons.org/licenses/by-nc-nd/3.0 